\title{
Portal Vein Embolization: What Do We Know?
}

\author{
Alban Denys · John Prior • Pierre Bize • \\ Rafael Duran · Thierry De Baere $\cdot$ Nermin Halkic • \\ Nicolas Demartines
}

Received: 11 July 2011/Accepted: 10 October 2011/Published online: 16 December 2011

(C) Springer Science+Business Media, LLC and the Cardiovascular and Interventional Radiological Society of Europe (CIRSE) 2011

\begin{abstract}
Portal vein embolization (PVE) has been developed to increase the size of the future remnant liver (FRL) left in place after major hepatectomy, thus reducing the risk of postoperative liver insufficiency. PVE consist in embolizing preoperatively portal branches of the segments that will be resected. Indication is based on preoperative measurements of the FRL by computed tomography and its ratio with either the theoretical liver volume or by direct measurement of the functional liver volume. After PVE, the volume and function of the FRL increases in 3 to 6 weeks, permitting extensive resections in patients otherwise contraindicated for liver resection. The PVE technique is variable from one center to another; however $n$-butyl-cyano-acrylate provides an interesting compromise between hypertrophy rate and procedure risk.
\end{abstract}

Keywords Embolization - Embolotherapy · Interventional oncology $\cdot$ Portal vein

\footnotetext{
A. Denys $(\bowtie) \cdot$ P. Bize $\cdot$ R. Duran

Department of Radiology and Interventional Radiology Unit, Centre Hospitalo Universitaire Vaudois, 41 rue du Bugnon, 1011 Lausanne, Switzerland

e-mail: Alban.Denys@chuv.ch

J. Prior

Department of Nuclear Medicine, Centre Hospitalo Universitaire Vaudois, 41 rue du Bugnon, 1011 Lausanne, Switzerland

T. De Baere

Department of Interventional Radiology, Institut Gustave Roussy, Pavillon de Recherche 1, 94805 Villejuif, France

N. Halkic $\cdot$ N. Demartines

Department of Visceral Surgery, Centre Hospitalo Universitaire

Vaudois, 41 rue du Bugnon, 1011 Lausanne, Switzerland
}

\section{Introduction}

Surgical resection of hepatic tumors is often the only curative option in primary and secondary liver tumors that may give patients a chance of long-term survival. However, the disease of many patients is considered unresectable because of an insufficient future remnant liver (FRL) volume to be left in place after extended surgical resection. The risk of liver failure after resection, as well as overall postoperative morbidity are directly linked to the volume of liver left in place by the surgeon [1]. In order to render more cases amenable to curative resection, portal vein embolization (PVE) is now an accepted technique to preoperatively increase the volume of the FRL.

PVE appeared in the late 1980s in Japan. In 1986, two publications opened the gate for the development of this technique. The first consisted of observations of hepatic lobar atrophy due to lobar portal invasion by cholangiocarcinoma [2]; the other consisted of a Japanese group's observations of atrophy of hepatic lobes in which they embolized portal branches in order to limit intraportal extension of hepatocellular carcinoma [3]. Simultaneously, they both noticed than segments with patent portal branches increased in size over time. These clinical observations confirmed the experimental results obtained by Rous and Larimore in the 1920s in a rabbit model of portal vein ligation [4]. The technique of PVE gained rapidly popularity in Japan in surgical groups treating hepatocellular carcinoma and Klatskin tumors [2, 3, 5, 6]. The first group that used PVE outside Japan was the Institut Gustave Roussy group in Villejuif, France, with Thierry de Baere as the interventional radiologist and Dominique Elias as the liver surgeon [7]. The first report in North America was that of David Madoff and Nicolas Vauthey from the MD Anderson Cancer Center in Houston, Texas, in 2000 [8]. 
Most liver surgeons have now endorsed this technique and use it in daily practice.

\section{How Does PVE Work?}

PVE is used in patient candidates who require extensive liver resection but have insufficient volume of FRL. Basically, PVE consist in occluding portal branches of segments that will be resected; the portal flow is then abruptly entirely redistributed toward the FRL's portal branches [9]. The mechanism of liver regeneration after PVE is a complex phenomenon that is not fully understood. From a histologic point of view, PVE induces atrophy of the embolized lobe as a result of both hepatocyte apoptosis and sinusoid dilatation, while in the nonembolized lobe, cells enter in an intense mitotic activity a few days after PVE, thus accounting for increased FRL volume after 2-4 weeks [10].

The biologic and cellular mechanisms of liver regeneration have been studied mainly in rodent models and in humans after extensive hepatectomy. From these publications, we know that hepatocytes that are usually quiescent in the G0 phase (only 1 out of 2000-3000 hepatocytes replicate in normal conditions) enter phase G1 one day after hepatectomy. Kupffer cells, endothelial cells, and bile duct cells replicate in a delayed fashion, suggesting that hepatocyte replication triggers proliferation of other cells [11]. After this initial phase of replication, hepatocytes increase progressively in size, allowing for gross liver growth [12]. Many molecular pathways are involved in liver regeneration after hepatectomy, such as tumor necrosis factor alpha, interleukin (IL) 6 produced by Kupffer cells, hepatocyte growth factor produced by stellate cells, vascular endothelial growth factor, and plateletderived growth factor [13]. Platelets and serotonin have also demonstrated their crucial role in liver regeneration [14]. All these factors interact and overlap in their activities and roles. Regeneration after PVE is somewhat different in term of intensity, with lower production of IL-6, IL-1, and tumor necrosis factor alpha after embolization. Mechanisms of regeneration after PVE are also delayed compared to regeneration after hepatectomy [15].

A deeper understanding of the mechanism involved in liver regeneration is crucial to improve the results of PVE. The role of heat shock protein 70 (a protein involved in cell reparation mechanisms) has also been evaluated as inductor of liver regeneration in human [16]. Miyake et al. have shown an increase by two- or fourfold in the nonembolized liver compared to embolized liver after PVE. Interestingly, in their series, only one patient did not show increase of heat shock protein 70 after PVE and subsequently died of liver failure after hepatectomy. Some groups have even explored the potential of heat shock protein 70 inducer geranyl-geranyl-acetone to stimulate liver regeneration. Administration of geranyl-geranyl-acetone increases tolerance to major hepatectomy but has never been tested in combination to PVE [17].

Despite many interesting animal and experimental studies, the trigger of liver regeneration after PVE remains unknown. The players are similar to those after hepatectomy, but the initial phenomenon remains mysterious. Is it a vascular stress in the FRL induced by abrupt portal redistribution, or is it in the embolized liver that the process is initiated by periportal inflammation? They remain open questions. It is noteworthy that different studies identified periportal inflammation in the embolized lobe from pathologic studies as an important predictor of liver regeneration, both in human and experimental animal studies [18, 19].

\section{How Are Patients Selected for PVE?}

Selection of patients for PVE is decided during multidisciplinary meetings and by tumor boards. The decision directly depends on the planning of the surgery, the amount of liver to be resected, and the functional status of the liver. This risk is related to the volume of liver left in place after surgery, to the function of the parenchyma (cirrhotic, cholestatic, fibrotic, steatotic) [12], and to the complexity of surgery that will prolong liver ischemia periods by vessel clamping and will increase intraoperative blood loss, and consequently will further increase risk of postoperative liver failure. Therefore, selection for PVE is based on three factors: liver volumetry as assessed by computed tomography (CT), liver function test, and resection complexity. Such ambitious surgical multistep projects are usually proposed to motivated patients in good general condition.

The first factor is easily established by means of CT after injection of contrast media. Attention should be paid to having a sufficient enhancement of both portal branches and hepatic veins to precisely delimit liver segments. Volumes are then delineated by manually tracking the limits of segments and tumors and automatically calculating volumes from axial slices. Recently, automatic recognition of liver segments allowing for "automatic volumetry" have become available from some companies [20].

Different methods have been used to determine the ratio between the FRL and the total functional liver volume (FLR ratio). Differences are related to the definition of total FLR. Some authors use direct measurement of the total liver volume minus tumor volume by CT [21-23]. Others use a standardized evaluation of the normal liver volume in white subjects on the basis of a formula using the formula body surface area (total liver volume $\left(\mathrm{cm}^{3}\right)=-794.41+1267.28 \times$ body surface area $\left.\left(\mathrm{m}^{2}\right)\right)$ [24]. The last method is to express this ratio 
as a percentage of body weight $[25,26]$. Shah et al. [25], comparing these three ways to estimate the FLR ratio, found that the best method to estimate the risk of postoperative liver failure was the formula estimating liver volume from body surface area described by Vauthey et al. [24]. The situation is probably different in diseased or cirrhotic liver, but to our knowledge, this has not been evaluated. In such conditions, comparison of the FLR ratio threshold between one publication and another becomes complex and not very feasible (Table 1). The consequence is that some groups, for patients with normal liver, use a FLR threshold at 30\% and others at $20 \%$ to decide PVE [26, 27].

Evaluation of liver function is more complex and debated. Patients with cirrhosis have been extensively studied in that regard, while patients treated by chemotherapy or with steatotic livers have been less studied. In patients with cirrhosis, estimation of hepatectomy risk is a conjunction of three elements: Child-Pugh score, measurement of portal hypertension, and, in some groups, indocyanine green (ICG) test. The latter evaluates both global liver perfusion and biliary excretion by measuring the extraction from the serum by the liver of ICG that is excreted unchanged into the bile. An ICG retention rate of $>20 \%$ at $15 \mathrm{~min}$ is considered to be a contraindication for resection [28]. Portal hypertension can be estimated by direct measurement of hepatic vein pressure gradients, by oesogastric endoscopy, or by imaging identification of large porto-caval collaterals [29, 30]. Selection criteria for other patients at high surgical risk are less established. For instance, there is no universally accepted way to select in patients treated by multiple courses of chemotherapy or with metabolic syndrome and steatosis [31, 32].

Table 1 Schematic description of the advantages and disadvantages of ipsilateral and contralateral approaches for PVE

\begin{tabular}{|c|c|c|}
\hline $\begin{array}{l}\text { Pros } \\
\text { and } \\
\text { cons }\end{array}$ & Contralateral & Ipsilateral \\
\hline \multirow[t]{3}{*}{ Pros } & Catheterism easier & No risk for FRL \\
\hline & $\begin{array}{l}\text { Final control } \\
\text { portography } \\
\text { easier }\end{array}$ & $\begin{array}{l}\text { Easy puncture as a result of larger } \\
\text { portal branches }\end{array}$ \\
\hline & Use of NBCA & Access to segment 4 branches \\
\hline \multirow[t]{5}{*}{ Cons } & $\begin{array}{l}\text { Risk of } \\
\text { complications in } \\
\text { the FRL }\end{array}$ & $\begin{array}{l}\text { Catheterism of right portal branches } \\
\text { more complex }\end{array}$ \\
\hline & \multirow{4}{*}{$\begin{array}{l}\text { (increased by } \\
\text { portal } \\
\text { hypertension) }\end{array}$} & Use of NBCA more tricky \\
\hline & & $\begin{array}{l}\text { Final control hard to achieve is } \\
\text { NBCA used }\end{array}$ \\
\hline & & Risk of tumor seeding \\
\hline & & $\begin{array}{l}\text { Risk of liver infarction in case of } \\
\text { arterial complication during access }\end{array}$ \\
\hline
\end{tabular}

Performing PVE in every patient before major resection does not influence postoperative morbidity and mortality [33]. The usual threshold accepted by most of the surgical teams is as follows. In young patients with a normal liver and without risk factors for liver surgery, a hepatectomy removing $75-80 \%$ of the functional liver is acceptable. In cirrhotic Child A patients with a portocaval gradient under $12 \mathrm{~mm} \mathrm{Hg}$, removing more than $60 \%$ of the liver volume is considered to put the patient at risk of postoperative liver failure. In patients with multiple courses of chemotherapy, steatosis, and cholestatic liver, and a FRL to total liver ratio of $20-40 \%$, the decision is made on an individual, basis taking all risk factors into consideration. A very small left lobe (under 10\%) should not be considered a contraindication; two recent studies have demonstrated in metastatic patients and in cirrhotic patients that there is a correlation between small initial size of the FLR and high degree of hypertrophy [23, 34]. In other words, the smaller the left lobe, the greater its hypertrophy after PVE.

\section{Should We Evaluate Liver Volumes or Liver Volume and Function by Nuclear Medicine?}

Over the last few decades, scintigraphic techniques have been used for noninvasive, direct evaluation of liver function and have several benefits over the more established, indirect method using CT volumetry [35].

${ }^{99 \mathrm{~m}}$ Tc-galactosyl human serum albumin $\left({ }^{99 \mathrm{~m}} \mathrm{Tc}-\mathrm{GSA}\right)$ scintigraphy measures the binding of asialoglycoproteins on its receptor, which is expressed only on the sinusoidal surface juxtaposing the Disse space of the mammalian hepatocytes [36]. The receptor is involved in the endocytosis of the asialoglycoproteins subsequently degraded by lysozymes. Over last few decades, several indices of liver function have been developed in planar scintigraphy and single-photon emission computed tomography (SPECT) [35]. They show a good correlation with conventional liver function tests, ICG clearance test, Child-Pugh classification, and histology (hepatic index activity score). In 9-17\% of patients, there is a discrepancy between ICG clearance testing and ${ }^{99 \mathrm{~m}} \mathrm{Tc}-\mathrm{GSA}$ scintigraphy; the latter better reflects the histologic severity of liver function $[37,38]$ and is not parasitized by hyperbilirubinemia.

Moreover, it is an independent predictor of postoperative complications, in contrast to ICG. Concerning PVE, ${ }^{99 \mathrm{~m}}$ Tc-GSA SPECT scintigraphy demonstrated additional value over $\mathrm{CT}$ volumetry for evaluating functional increase after PVE [39-41], by demonstrating a higher increase in function of the nonembolized liver by ${ }^{99 \mathrm{~m}} \mathrm{Tc}-\mathrm{GSA}$ SPECT/ CT than by CT-volumetry $(+21.4 \%$ vs. $+13.9 \%$, $P<0.001)$. In a similar study using ${ }^{99 \mathrm{~m}}$ Tc-GSA SPECT/ $\mathrm{CT}$, criteria could be proposed on the basis of total amount 
of receptor in the remnant liver to select candidates for PVE with good clinical outcome and thus expand the range of hepatic resection [42]. Unfortunately, the ${ }^{99 \mathrm{~m}} \mathrm{Tc}-\mathrm{GSA}$ kit is only commercially available in Japan and is not currently available for use in Europe or the United States (Fig. 1).

The ${ }^{99 \mathrm{~m}}$ Tc-iminodiacetic acid $\left({ }^{99 \mathrm{~m}}\right.$ Tc-IDA) derivative scintigraphy has been used for more than three decades for hepatobiliary scintigraphy. These lidocaine analogs are transported to the liver mainly bound to albumin, where it is cleaved in the Disse space. From there, ${ }^{99 m}$ Tc-IDA compounds enter the basal membrane of the hepatocyte through anion transporters before being excreted unmetabolized to the biliary tract [43] — similar to ICG-by the ATP-dependent export pump multidrug-associated protein 2. As these agents follow a path similar to bilirubin or toxins, they have been proposed as an index of liver function [44]. Of all available IDA derivatives, ${ }^{99 \mathrm{~m}} \mathrm{Tc}$-mebrofenin is the agent of choice, with high hepatic uptake, minimal urinary excretion, and resistance to high levels of bilirubinemia. Hybrid SPECT/CT acquisitions have been used to derive regional liver function around the peak of the hepatic time-activity curve, allowing calculation of remnant liver function on the basis of contouring the liver outline via low-dose CT [45].

${ }^{99} \mathrm{~m}$ Tc-mebrofenin scintigraphy has been validated in the preoperative assessment of liver function [46, 47], with a good correlation with ICG testing [48]. The combination of dynamic hepatobiliary scintigraphy and SPECT to morphologic volume measured by CT was able to accurately predict actual postoperative liver function of the remnant liver [45]. This technique is currently applied to measure the regional increase in liver function after PVE. ${ }^{99 \mathrm{~m}} \mathrm{Tc}-$ mebrofenin scintigraphy could be used to select patients for PVE thanks to its demonstrated ability to predict increased postoperative liver failure [49]. A step in this direction was taken using hypothetical values for safe resection in function and volume increase after PVE. De Graaf et al. [50] showed that the increase in function as measured by ${ }^{99 \mathrm{~m}} \mathrm{Tc}$ mebrofenin was larger than the increase in volume. This suggests that the waiting time until resection may be shorter than the 3-4 weeks indicated by volume expansion. Thus, some authors advocate that function-based criteria, in addition to volume-based criteria, should be used, especially when liver resection needs to be performed with minimal accepted remnant volume [51].

\section{How Is PVE Performed?}

The technique of PVE is extremely variable from one center to another, depending on operator preference (Table 1). The access route can be ipsilateral or contralateral. Some authors use the ipsilateral approach, puncturing a right portal branch and embolizing in a retrograde fashion all right portal branches. This access allows for an easy catheterization of segment 4 branches when they must be embolized. The drawback of this technique is mainly the difficulty of access to the right portal branches in a retrograde fashion, and also sometimes the difficulty of finding a route through healthy liver to the right portal branches $[6,52]$. The contralateral approach aims to puncture a left peripheral portal branch (Fig. 1). Catheterization of the right portal branches is theoretically easier, if anatomy is standard. Such contralateral access renders final control portography easier because the catheter does not have to pass through embolic material to be placed in the portal vein for final contrast injection [7, 23, 53]. Choosing the access also depends on the embolic material used. Glue can hardly be manipulated from the ipsilateral side, while large embolic materials, like plugs, need large-diameter access, which is less risky when obtained on the ipsilateral side [54]. The final choice between the ipsilateral and contralateral routes should be made by comparing their respective complication rates. They seem similar and are mainly related to puncture of unexpected structures, such as biliary branches or hepatic arteries. The largest series of contralateral PVE reviewed 188 cases at different centers using contralateral access and $n$-butyl-cyanoacrylate as an embolic material [55]. Only six of the 12 reported
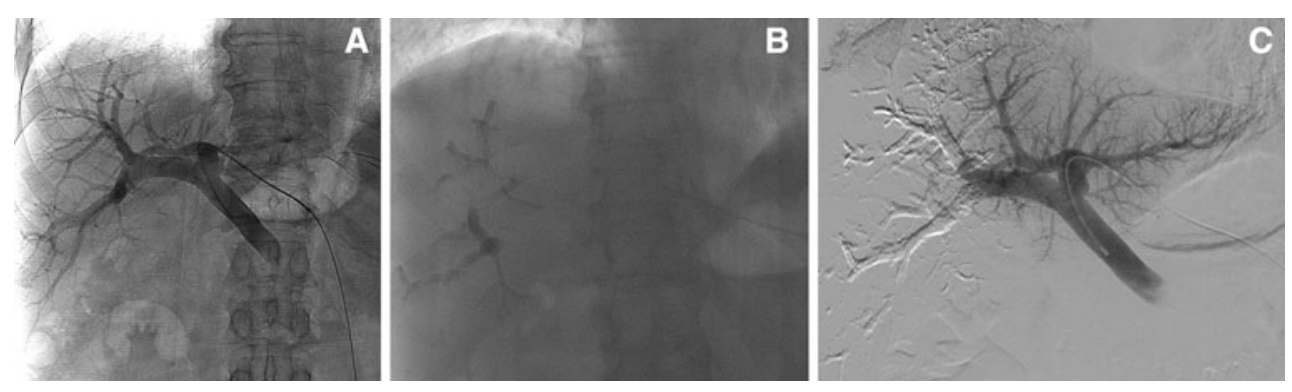

Fig. 1 A 67-year-old man bearing four liver metastases in the right lobe. The decision was made to perform PVE before right hemihepatectomy. A Segment 3 branch and portography in the right anterior oblique view were obtained. B The right portal branches were embolized with a mixture of NBCA and ethiodized oil. C Subtracted portography after PVE showing complete redistribution toward the left lobe. Parenchymatous enhancement was only evident on the right side 
complications could be related to the access route, but none precluded liver resection. It is also interesting to note that these complications mainly occurred in patients with portal hypertension. Another series reported similar rate of complications using the ipsilateral route [56]. Finally, arterial or biliary complications do not have the same consequences if the portal vein is occluded or patent. Embolization of a traumatized hepatic arterial branch may lead to infarction on the ipsilateral side of PVE and have no impact on the contralateral side. Our policy for right PVE using n-butyl-cyano-acrylate (NBCA) is to use the contralateral side. We use the ipsilateral side when segment 4 branches must be embolized and when the left lobe is quite small and barely accessible. Other access routes have been described through surgical dissection and catheterization of the ileocolic vein. This access has the benefit of not puncturing the liver, but it makes the procedure more complex and catheter manipulation trickier [57].

Our recommendation and habit is to use the contralateral access route and to embolize with NBCA mixed with lipiodol. The risk of local complication is low and can be managed without difficulty because the portal flow is opened on this side (Fig. 1).

\section{Which Embolic Material Should Be Used?}

Various embolic materials have been used for PVE, including Gelfoam [7], NBCA [23], different types and size of beads [58, 59], alcohol [60], and nitinol plugs [54] (Table 2). All of these embolic materials are able to occlude the right portal branches and redistribute flow toward the FRL. There is no official recommendation for a specific embolic material [61]. There is no single-center comparative study evaluating which embolic material provide better hypertrophy, and comparison between series are not helpful because these series have neither standardized inclusion criteria nor standardized delay after PVE for CT evaluation of hypertrophy (2-6 weeks). In addition, the rate of hypertrophy after PVE depends on the preembolization FRL volume more than any other factor, as described previously [34].

However, the choice of the embolic material can be influenced by the results of experimental studies. Recently, De Baere et al. have compared in a pig model three commercially available embolic materials: NBCA mixed with iodized oil, and two different sizes of spherical particles. NBCA seemed to be more efficient in including liver regeneration than spherical particles. A correlation of periportal fibrosis in the embolized lobe and liver regeneration was also found [19]. This result is in concordance with previous observations in human. If particles are used, more significant hypertrophy is obtained with small-size spherical particles compared to larger-size or nonspherical particles [62].

\section{Should PVE or Surgical Ligation of the Right Portal Branches Be Performed?}

Surgical ligation of the right portal branches is an invasive surgical procedure requiring dissection of the liver hilum during a laparotomy. Controversy exists about the respective indications of PVE and ligation. Portal vein ligation seems efficient to induce left lobe hypertrophy [63, 64], but because of its invasiveness, it is mainly used in the so-called two-stage hepatectomy [65]. Patients with bilobar metastases are operated on for resection of left lobe metastases, and right portal vein ligation is achieved in the same procedure. Four to 5 weeks thereafter, the left liver has increased in size, and right hepatectomy can be performed. However, portal vein ligation does not occlude distal portal branches; the development of multiple intrahepatic porto-portal collaterals-namely from segment 4 to segments 5 and 8 -is possible [66]. Even if portal vein ligation allows for a two-stage hepatectomy, another option in bilobar disease with small-size tumor in the left is radiofrequency ablation of the left liver metastases and PVE in the same procedure [67], followed 4 weeks later by right hemihepatectomy.

Animal studies have conflicting results comparing regeneration rate after portal vein ligation or embolization. Studies in rats demonstrated superiority of ligation [68], while more recent studies have shown the opposite in larger animals (pigs and rabbits) [69, 70]. The explanation might be that the liver in rodents are almost foliated with separated liver segments, while in pigs and in rabbits intrahepatic porto-portal collaterals developed, thus probably limiting the occlusive effect of ligation. Furthermore, hilar dissection in rats is probably associated by arterial lesions in the ligated segments, increasing the effect of portal ligation.

\section{Should Segment 4 Branches Be Embolized?}

In a prospective study analyzing liver volumes in a normal population [71], volumes of segments 2 and 3 have shown to account for less than $20 \%$ of the total liver volume in nearly $80 \%$ of the population. In other words, PVE should be performed in $80 \%$ of the cases when an extended right hepatectomy has to be performed. The question is in these cases is, should we or should we not embolize segment 4 branches? The results described in the literature are controversial. Two elements should be kept in mind when evaluating patients for segment 4 embolization. First, the procedure is much more complex and can hardly performed from the contralateral approach. This implies that NBCA will probably not be used, and that a very careful embolization will be performed on these branches with 


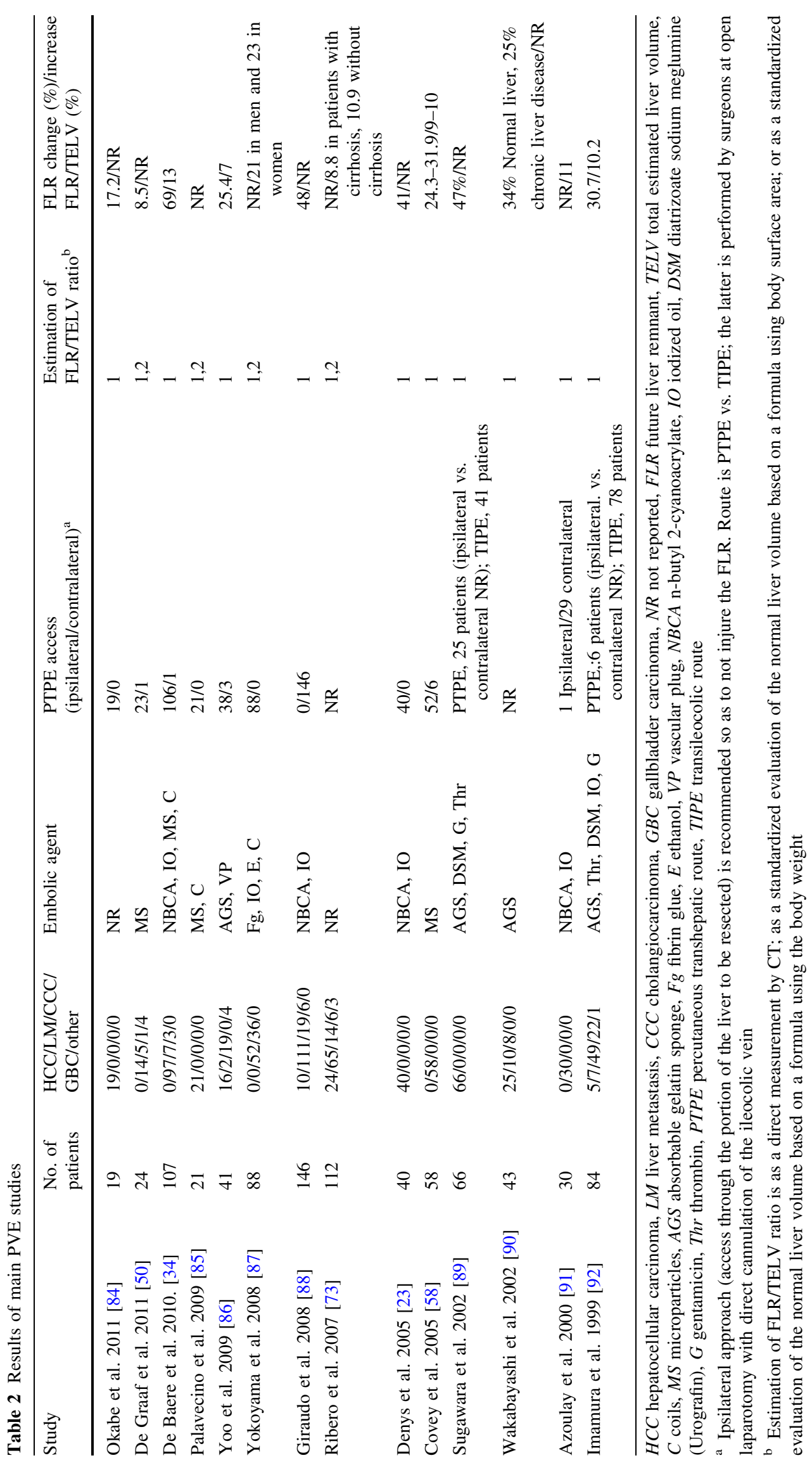


particles and coils. This also means that the surgeon will have to resect segment 4 in toto, which is rarely the case in clinical practice. Because segment 4 has multiple portal branches, it can also be resected incompletely, leaving more parenchyma postoperatively.

Madoff et al. initially reported that embolization of segment 4 branches nearly double the rate of hypertrophy of the left lobe [62]. These results have not been found in other experienced centers, which have even observed the contrary $[34,72]$. These groups observed maintained hypertrophy of segment 4 after embolization of segment 4 branches. They suggested that segment 4 portal-branch embolization is rarely complete, and that persistent patent segment 4 portal branches account for maintained regeneration.

\section{What Should Be Done If Hypertrophy Is Insufficient after PVE?}

This situation is more frequent when considering patients with chronic liver disease. Indeed, in a retrospective analysis of 42 consecutive cases, we observed nearly $10 \%$ of patients without significant hypertrophy after PVE [23]. These patients were not operated on because insufficient hypertrophy after PVE is usually considered a risk factor for liver insufficiency after PVE. For some authors, a degree of hypertrophy estimated as a percentage of increase of the FRL below $10 \%$ in patients having chronic liver disease is an indication of high risk of liver insufficiency [33]; the same was observed with a degree of hypertrophy of $5 \%$ for patients with normal liver [73]. Associated risk factors for liver insufficiency in patients after hepatectomy prepared by PVE are associated jaundice and extensive dissection of the hepaticoduodenal ligament [74].
In cases of patients with chronic liver disease, it may be necessary to wait longer (6-8 weeks after PVE) to obtain sufficient hypertrophy. A recent study has demonstrated that hypertrophy and regeneration may continue over a 1-year period after PVE [75].

\section{Can We Predict Liver Regeneration after PVE?}

There is no way to predict hypertrophy after PVE. Many factors have been identified and influence regeneration. However, the most significant factor is the size of the FRL before PVE [23, 34], in cases of both healthy liver and chronic liver disease. This means that surgeons and interventionalist should not preclude PVE in case of very small left liver volumes, but on the contrary expect marked hypertrophy in these cases. Indeed, PVE was able to provide enough hypertrophy to convert the patient to surgery with a FRL as low as $6.9 \%$, which clearly open the gate for resection of all the liver except one segment-a notion that to our knowledge has never been explored. Other factors identified as decreasing the rate of regeneration must be kept in mind, and in these cases, PVE can be considered as a test for the capacity of the liver to regenerate. Factors known to negatively influence regeneration include diabetes, liver fibrosis F4, cholestasis, and portal hypertension. Other factors are known to have no influence on liver regeneration, including sex age, origin of the tumor, chemotherapy with anti-vascular endothelial growth factor such as bevacizumab, and ICG clearance [76]. Chemotherapy with oxaliplatin may induce severe sinusoidal obstruction and subsequent portal hypertension, making PVE more difficult and potentially more at risk of complications [77] (Fig. 2).
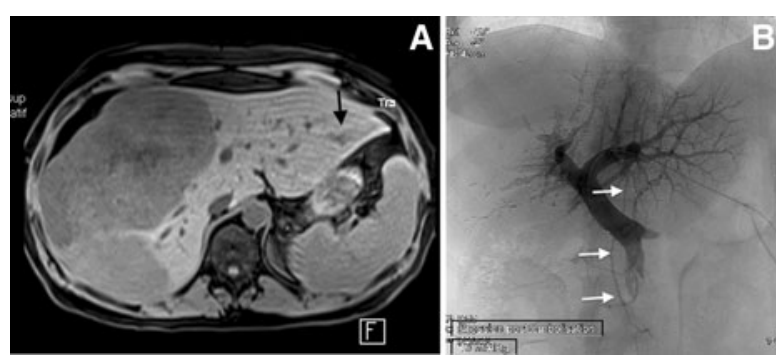

Fig. 2 A 45-year-old woman treated with six cycles of chemotherapy with oxaliplatin, 5-fluorouracil, and folinic acid. Because of the presence of stable disease, right hepatectomy was chosen. Because FRL ratio is $28 \%$, and taking into account the percutaneous destruction of a small segment 3 lesion, a right PVE was performed. A T1-weighted axial image identifying both right hepatic lobe metastases and small nodular lesion in segment 3 (arrow). B After PVE, portography confirmed occlusion of right portal branches, and
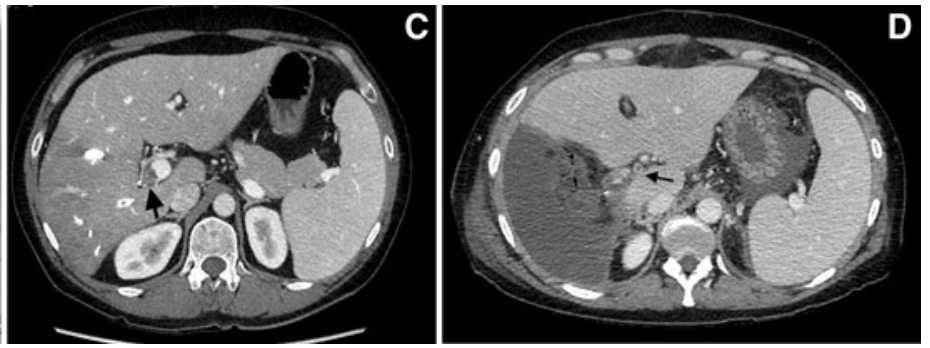

the hepatofugal paraumbilical vein was identified (white arrows). C A control CT was performed 1 month after PVE, revealing partial thrombosis of the portal vein trunk (arrow), while segments 2, 3, and 4 increased in size. D During hepatectomy, right portal vein ligation was made more complex by portal vein thrombosis and stenosis of the portal vein associated with portal hypertension after hepatectomy induced complete portal vein thrombosis at postoperative CT (arrow) 


\section{Is Surgery More Complex after PVE?}

Complications after PVE should not be underestimated and may impair future surgery. Hematoma, hemobilia, and sepsis, as well as embolization material going to the nonembolized lobe, resulting in partial or complete portal vein thrombosis, are rare but may be serious problems for surgery [34]. For surgical strategy in cases of hilar cholangiocarcinoma, it is important to preoperatively precisely determine the liver side to be embolized and resected. It is obvious that once the PVE is performed, an intraoperative change in the resection strategy is no longer possible, thus making a preoperative precise diagnosis mandatory before PVE [78].

Even without PVE-induced complications, postoperative complications of major liver surgery after PVE are increased, with a clear trend for higher intraoperative bleeding from the dilated intrahepatic venous collateral [34, 79-81].

In fact, operation duration after PVE is significantly longer, and there is increased blood loss. The postoperative complication rate after PVE is about $40 \%$, with mortality at 30,60 , and 90 days of $2 \%, 4.7 \%$, and $6 \%$, respectively [81]. This increased morbidity and blood loss are due in part to more complex resection, but also to the abovementioned dilated collateral veins after PVE. The important point is that blood loss has been correlated with impaired postoperative liver regeneration in an experimental model [82]. This is correlated with clinical studies that reveal blood transfusion to be an independent predictive factor for postoperative liver insufficiency [81, 83].

Major postoperative changes in portal blood flow after PVE followed by extensive liver resection are observed. The volume of portal blood flow may increase up to threefold and may lead to a relative venous outflow block and liver congestions, thus forming a small-for-size liver [78].

\section{Conclusion}

PVE is a well-established technique. It is now used worldwide to enhance patient safety after major hepatectomy. This technique is probably still in its infancy; regeneration enhancers, safer embolic material, association to hepatic vein embolization are many new ways that will permit more aggressive surgical options. Removing all the liver except for one segment is still a utopian ideal-but probably not for long.

Conflict of interest The authors declare that they have no conflict of interest.

\section{References}

1. Vauthey JN, Baer HU, Guastella T et al (1993) Comparison of outcome between extended and nonextended liver resections for neoplasms: a multivariate analysis of prognostic factors. Surgery 114:968-975

2. Takayasu K, Muramatsu Y, Shyma Y et al (1986) Hepatic lobar atrophy following obstruction of the ipsilateral portal vein from hilar cholangiocarcinoma. Radiology 160:389-393

3. Kinoshita H, Sakai K, Hirohashi K et al (1986) Preoperative portal vein embolization for hepatocellular carcinoma. World $\mathrm{J}$ Surg 10:803-808

4. Rous P, Larimore L (1920) Relation of portal blood to liver maintenance. J Exp Med 31:609-632

5. Makuuchi M, Kosugue T, Lygidakis N (1991) New possibilities for major liver surgery in patients with Klatskin tumors or primary hepatocellular carcinoma-an old problem revisited. Hepatogastroenterology 38:329-336

6. Makuushi M, Le Thai B, Takayasu K et al (1990) Preoperative portal embolization to increase safety of major hepatectomy for hilar bile duct carcinoma: a preliminary report. Surgery 107:521-527

7. de Baere T, Roche A, Vavasseur D et al (1993) Portal vein embolization: utility for inducing left hepatic lobe hypertrophy before surgery. Radiology 188:73-77

8. Abdalla EK, Barnett CC, Doherty D et al (2002) Extended hepatectomy in patients with hepatobiliary malignancies with and without preoperative portal vein embolization. Arch Surg 137: 675-680

9. Denys AL, Abehsera M, Leloutre B et al (2000) Intrahepatic hemodynamic changes following portal vein embolization: a prospective Doppler study. Eur Radiol 10:1703-1707

10. Harada H, Imamura H, Miyagawa S, Kawasaki S (1997) Fate of the human liver after hemihepatic portal vein embolization: cell kinetic and morphometric study. Hepatology 26:1162-1170

11. Michalopoulos GK, De Frances MC (1997) Liver regeneration. Science 276(5309):60-66

12. Clavien PA, Petrowsky H, DeOliveira ML, Graf R (2007) Strategies for safer liver surgery and partial liver transplantation. N Engl J Med 356:1545-1559

13. Clavien PA (2008) Liver regeneration: a spotlight on the novel role of platelets and serotonin. Swiss Med Wkly 138:361-370

14. Clavien PA, Graf R (2009) Liver regeneration and platelets. Br J Surg 96:965-966

15. Takamura K, Tsuchida K, Miyake H et al (2005) Activin and activin receptor expression changes in liver regeneration in rat. J Surg Res 126:3-11

16. Miyake H, Fujii M, Sasaki K et al (2003) Heat shock protein 70 induction in hepatocytes after right portal vein embolization. Hepatogastroenterology 50:2084-2087

17. Oda H, Miyake H, Iwata T et al (2002) Geranylgeranylacetone suppresses inflammatory responses and improves survival after massive hepatectomy in rats. J Gastrointest Surg 6:464-472

18. Tanaka H, Hirohashi K, Kubo S et al (1999) Influence of histological inflammatory activity on regenerative capacity of liver after percutaneous transhepatic portal vein embolization. J Gastroenterol 34:100-104

19. de Baere T, Denys A, Paradis V (2009) Comparison of four embolic materials for portal vein embolization: experimental study in pigs. Eur Radiol 19:1435-1442

20. Zhou JY, Wong DW, Ding F et al (2010) Liver tumour segmentation using contrast-enhanced multi-detector CT data: performance benchmarking of three semiautomated methods. Eur Radiol 20:1738-1748 
21. Azoulay D, Castaing D, Krissat J et al (2000) Percutaneous portal vein embolization increases the feasibility and safety of major liver resection for hepatocellular carcinoma in injured liver. Ann Surg 232:665-672

22. de Baere T, Denys A, Madoff DC (2007) Preoperative portal vein embolization: indications and technical considerations. Tech Vasc Interv Radiol 10:67-78

23. Denys A, Lacombe C, Schneider F et al (2005) Portal vein embolization with N-butyl cyanoacrylate before partial hepatectomy in patients with hepatocellular carcinoma and underlying cirrhosis or advanced fibrosis. J Vasc Interv Radiol 16:1667-1674

24. Vauthey JN, Abdalla EK, Doherty DA et al (2002) Body surface area and body weight predict total liver volume in Western adults. Liver Transpl 8:233-240

25. Shah A, Goffette P, Hubert C et al (2011) Comparison of different methods to quantify future liver remnants after preoperative portal vein embolization to predict postoperative liver failure. Hepatogastroenterology 58:109-114

26. Vauthey JN, Chaoui A, Do KA et al (2000) Standardized measurement of the future liver remnant prior to extended liver resection: methodology and clinical associations. Surgery 127: 512-519

27. Elias D, Ouellet JF, De Baere T et al (2002) Preoperative selective portal vein embolization before hepatectomy for liver metastases: long-term results and impact on survival. Surgery 131:294-299

28. Imamura H, Sano K, Sugawara Y et al (2005) Assessment of hepatic reserve for indication of hepatic resection: decision tree incorporating indocyanine green test. J Hepatobiliary Pancreat Surg 12:16-22

29. Bruix J, Castells A, Bosch J et al (1996) Surgical resection of hepatocellular carcinoma in cirrhotic patients: prognostic value of preoperative portal pressure. Gastroenterology 111:1018-1022

30. Poon RT, Fan ST (2005) Assessment of hepatic reserve for indication of hepatic resection: how I do it. J Hepatobiliary Pancreat Surg 12:31-37

31. Abdalla EK (2010) Portal vein embolization (prior to major hepatectomy) effects on regeneration, resectability, and outcome. J Surg Oncol 102:960-967

32. Farges O, Malassagne B, Flejou JF et al (1999) Risk of major liver resection in patients with underlying chronic liver disease: a reappraisal. Ann Surg 229:210-215

33. Farges O, Belghiti J, Kianmanesh R et al (2003) Portal vein embolization before right hepatectomy: prospective clinical trial. Ann Surg 237:208-217

34. de Baere T, Teriitehau C, Deschamps F et al (2010) Predictive factors for hypertrophy of the future remnant liver after selective portal vein embolization. Ann Surg Oncol 17:2081-2089

35. de Graaf W, Bennink RJ, Vetelainen R, van Gulik TM (2010) Nuclear imaging techniques for the assessment of hepatic function in liver surgery and transplantation. J Nucl Med 51:742-752

36. Vera DR, Krohn KA, Stadalnik RC, Scheibe PO (1984) Tc-99 mgalactosyl-neoglycoalbumin: in vivo characterization of receptormediated binding to hepatocytes. Radiology 151:191-196

37. Kwon AH, Ha-Kawa SK, Uetsuji S et al (1997) Preoperative determination of the surgical procedure for hepatectomy using technetium-99 m-galactosyl human serum albumin $\left({ }^{99 \mathrm{~m}} \mathrm{Tc}-\mathrm{GSA}\right)$ liver scintigraphy. Hepatology 25:426-429

38. Nanashima A, Yamaguchi H, Shibasaki S et al (2004) Relationship between indocyanine green test and technetium-99 m galactosyl serum albumin scintigraphy in patients scheduled for hepatectomy: clinical evaluation and patient outcome. Hepatol Res 28:184-190

39. Hirai I, Kimura W, Fuse A et al (2003) Evaluation of preoperative portal embolization for safe hepatectomy, with special reference to assessment of nonembolized lobe function with 99 mTc-GSA SPECT scintigraphy. Surgery 133:495-506

40. Nanashima A, Tobinaga S, Abo T et al (2010) Relationship of hepatic functional parameters with changes of functional liver volume using technetium- $99 \mathrm{~m}$ galactosyl serum albumin scintigraphy in patients undergoing preoperative portal vein embolization: a follow-up report. J Surg Res 164:e235-e242

41. Nishiyama Y, Yamamoto Y, Hino I et al (2003) 99mTc galactosyl human serum albumin liver dynamic SPET for pre-operative assessment of hepatectomy in relation to percutaneous transhepatic portal embolization. Nucl Med Commun 24:809-817

42. Yumoto Y, Yagi T, Sato S et al (2010) Preoperative estimation of remnant hepatic function using fusion images obtained by (99m)Tc-labelled galactosyl-human serum albumin liver scintigraphy and computed tomography. Br J Surg 97:934-944

43. Krishnamurthy GT, Krishnamurthy S (2006) Cholescintigraphic measurement of liver function: how is it different from other methods? Eur J Nucl Med Mol Imaging 33:1103-1106

44. Heyman S (1994) Hepatobiliary scintigraphy as a liver function test. J Nucl Med 35:436-437

45. de Graaf W, van Lienden KP, van Gulik TM, Bennink RJ (2010) (99m)Tc-mebrofenin hepatobiliary scintigraphy with SPECT for the assessment of hepatic function and liver functional volume before partial hepatectomy. J Nucl Med 51:229-236

46. Bennink RJ, Dinant S, Erdogan D et al (2004) Preoperative assessment of postoperative remnant liver function using hepatobiliary scintigraphy. J Nucl Med 45:965-971

47. van den Esschert JW, de Graaf W, van Lienden KP et al (2009) Volumetric and functional recovery of the remnant liver after major liver resection with prior portal vein embolization : recovery after PVE and liver resection. J Gastrointest Surg 13:1464-1469

48. Erdogan D, Heijnen BH, Bennink RJ et al (2004) Preoperative assessment of liver function: a comparison of $99 \mathrm{mTc}-M e b r o f e n i n$ scintigraphy with indocyanine green clearance test. Liver Int 24:117-123

49. Dinant S, de Graaf W, Verwer BJ et al (2007) Risk assessment of posthepatectomy liver failure using hepatobiliary scintigraphy and CT volumetry. J Nucl Med 48:685-692

50. de Graaf W, van Lienden KP, van den Esschert JW et al (2011) Increase in future remnant liver function after preoperative portal vein embolization. Br J Surg 98:825-834

51. Bennink RJ, de Graaf W, van Gulik TM (2011) Toward a total and regional scintigraphic liver function test enabling assessment of future remnant liver function. Nucl Med Commun 32:669-671

52. Madoff DC, Abdalla EK, Vauthey JN (2005) Portal vein embolization in preparation for major hepatic resection: evolution of a new standard of care. J Vasc Interv Radiol 16:779-790

53. De Baere T, Roche A, Elias D et al (1996) Preoperative portal vein embolization for extension of hepatectomy indications. Hepatology 24:1386-1391

54. Bent CL, Low D, Matson MB et al (2009) Portal vein embolization using a nitinol plug (Amplatzer vascular plug) in combination with histoacryl glue and iodinized oil: adequate hypertrophy with a reduced risk of nontarget embolization. Cardiovasc Intervent Radiol 32:471-477

55. Di Stefano DR, de Baere T, Denys A et al (2005) Preoperative percutaneous portal vein embolization: evaluation of adverse events in 188 patients. Radiology 234:625-630

56. Kodama Y, Shimizu T, Endo H et al (2002) Complications of percutaneous transhepatic portal vein embolization. J Vasc Interv Radiol 13:1233-1237

57. Madoff DC, Hicks ME, Vauthey JN et al (2002) Transhepatic portal vein embolization: anatomy, indications, and technical considerations. Radiographics 22:1063-1076 
58. Covey AM, Tuorto S, Brody LA et al (2005) Safety and efficacy of preoperative portal vein embolization with polyvinyl alcohol in 58 patients with liver metastases. AJR Am J Roentgenol $185: 1620-1626$

59. Madoff DC, Hicks ME, Abdalla EK et al (2003) Portal vein embolization with polyvinyl alcohol particles and coils in preparation for major liver resection for hepatobiliary malignancy: safety and effectiveness-study in 26 patients. Radiology 227 : 251-260

60. Ogasawara K, Uchino J, Une Y, Fujioka Y (1996) Selective portal vein embolization with absolute ethanol induces hepatic hypertrophy and makes more extensive hepatectomy possible. Hepatology 23:338-345

61. Denys A, Bize P, Demartines N et al (2010) Quality improvement for portal vein embolization. Cardiovasc Intervent Radiol 33: 452-456

62. Madoff DC, Abdalla EK, Gupta S et al (2005) Transhepatic ipsilateral right portal vein embolization extended to segment IV: improving hypertrophy and resection outcomes with spherical particles and coils. J Vasc Interv Radiol 16(21):215-225

63. Aussilhou B, Lesurtel M, Sauvanet A et al (2008) Right portal vein ligation is as efficient as portal vein embolization to induce hypertrophy of the left liver remnant. J Gastrointest Surg 12:297-303

64. Kianmanesh R, Farges O, Abdalla EK et al (2003) Right portal vein ligation: a new planned two-step all-surgical approach for complete resection of primary gastrointestinal tumors with multiple bilateral liver metastases. J Am Coll Surg 197:164-170

65. Jaeck D, Bachellier P, Nakano H et al (2003) One or two-stage hepatectomy combined with portal vein embolization for initially nonresectable colorectal liver metastases. Am J Surg 185:221-229

66. Denys AL, Abehsera M, Sauvanet A et al (1999) Failure of right portal vein ligation to induce left lobe hypertrophy due to intrahepatic portoportal collaterals: successful treatment with portal vein embolization. AJR Am J Roentgenol 173:633-635

67. Elias D, Santoro R, Ouellet JF et al (2004) Simultaneous percutaneous right portal vein embolization and left liver tumor radiofrequency ablation prior to a major right hepatic resection for bilateral colorectal metastases. Hepatogastroenterology 51: 1788-1791

68. Furrer K, Tian Y, Pfammatter T et al (2008) Selective portal vein embolization and ligation trigger different regenerative responses in the rat liver. Hepatology 47:1615-1623

69. van den Esschert JW, van Lienden KP, de Graaf W et al (2011) Portal vein embolization induces more liver regeneration than portal vein ligation in a standardized rabbit model. Surgery 149:378-385

70. Wilms C, Mueller L, Lenk C et al (2008) Comparative study of portal vein embolization versus portal vein ligation for induction of hypertrophy of the future liver remnant using a mini-pig model. Ann Surg 247:825-834

71. Abdalla EK, Denys A, Chevalier P et al (2004) Total and segmental liver volume variations: implications for liver surgery. Surgery 135:404-410

72. Kishi Y, Madoff DC, Abdalla EK et al (2008) Is embolization of segment 4 portal veins before extended right hepatectomy justified? Surgery 144:744-751

73. Ribero D, Abdalla EK, Madoff DC et al (2007) Portal vein embolization before major hepatectomy and its effects on regeneration, resectability and outcome. Br J Surg 94:1386-1394

74. Fujii Y, Shimada H, Endo I et al (2003) Risk factors of posthepatectomy liver failure after portal vein embolization. J Hepatobiliary Pancreat Surg 10:226-232
75. Correa D, Schwartz L, Jarnagin WR et al (2010) Kinetics of liver volume changes in the first year after portal vein embolization. Arch Surg 145:351-354

76. Covey AM, Brown KT, Jarnagin WR et al (2008) Combined portal vein embolization and neoadjuvant chemotherapy as a treatment strategy for resectable hepatic colorectal metastases. Ann Surg 247:451-455

77. Rubbia-Brandt L, Mentha G, Terris B (2006) Sinusoidal obstruction syndrome is a major feature of hepatic lesions associated with oxaliplatin neoadjuvant chemotherapy for liver colorectal metastases. J Am Coll Surg 202:199-200

78. van Gulik TM, van den Esschert JW, de Graaf W et al (2008) Controversies in the use of portal vein embolization. Dig Surg $25: 436-444$

79. Broering DC, Hillert C, Krupski G et al (2002) Portal vein embolization vs. portal vein ligation for induction of hypertrophy of the future liver remnant. J Gastrointest Surg 6:905-913

80. Hwang S, Lee SG, Ko GY et al (2009) Sequential preoperative ipsilateral hepatic vein embolization after portal vein embolization to induce further liver regeneration in patients with hepatobiliary malignancy. Ann Surg 249:608-616

81. Kishi Y, Abdalla EK, Chun YS et al (2009) Three hundred and one consecutive extended right hepatectomies: evaluation of outcome based on systematic liver volumetry. Ann Surg 250: $540-548$

82. Okano T, Ohwada S, Nakasone Y et al (2001) Blood transfusion causes deterioration in liver regeneration after partial hepatectomy in rats. J Surg Res 101:157-165

83. Halazun KJ, Al-Mukhtar A, Aldouri A et al (2007) Right hepatic trisectionectomy for hepatobiliary diseases: results and an appraisal of its current role. Ann Surg 246:1065-1074

84. Okabe H, Beppu T, Ishiko T et al (2011) Preoperative portal vein embolization (PVE) for patients with hepatocellular carcinoma can improve resectability and may improve disease-free survival. J Surg Oncol 104:641-646

85. Palavecino M, Chun YS, Madoff DC et al (2009) Major hepatic resection for hepatocellular carcinoma with or without portal vein embolization: perioperative outcome and survival. Surgery 145: 399-405

86. Yoo H, Ko GY, Gwon DI et al (2009) Preoperative portal vein embolization using an amplatzer vascular plug. Eur Radiol 19:1054-1061

87. Yokoyama Y, Nagino M, Oda K et al (2008) Sex dimorphism in the outcome of preoperative right portal vein embolization. Arch Surg 143:254-259

88. Giraudo G, Greget M, Oussoultzoglou E et al (2008) Preoperative contralateral portal vein embolization before major hepatic resection is a safe and efficient procedure: a large single institution experience. Surgery 143:476-482

89. Sugawara Y, Yamamoto J, Higashi H et al (2002) Preoperative portal embolization in patients with hepatocellular carcinoma. World J Surg 26:105-110

90. Wakabayashi H, Ishimura K, Okano K et al (2002) Application of preoperative portal vein embolization before major hepatic resection in patients with normal or abnormal liver parenchyma. Surgery 131:26-33

91. Azoulay D, Castaing D, Smail A et al (2000) Resection of nonresectable liver metastases from colorectal cancer after percutaneous portal vein embolization. Ann Surg 231:480-486

92. Imamura H, Shimada R, Kubota M et al (1999) Preoperative portal vein embolization: an audit of 84 patients. Hepatology 29:1099-1105 